\title{
Extensive phlegmon and pyoderma gangrenosum: diagnostic difficulties
}

\author{
Dominika Wcisło-Dziadecka1, Beata Bergler-Czop², Ligia Brzezińska-Wcisło², Hubert Arasiewicz²
}

${ }^{1}$ Medical Univesity of Silesia, School of Medicine in Katowice, Department of Cosmetology, Institute of Structural Research of Skin, Katowice, Poland

Head of Department: Krzysztof Jasik PhD

${ }^{2}$ Medical Univesity of Silesia, School of Medicine in Katowice, Chair and Department of Dermatology, Katowice, Poland Head of Department: Prof. Ligia Brzezińska-Wcisło MD, PhD

Postep Derm Alergol 2015; XXXII, 1: 46-50

DOI: $10.5114 /$ pdia.2014.40947

\begin{abstract}
Pyoderma gangrenosum (PG) is a relatively rare neutrophilic dermatosis, characterized by progressive skin necrosis. It typically has a chronic course, of unknown etiology. Pyoderma gangrenosum diagnosis can be difficult because both histopathological examination and results of additional laboratory tests are not specific and the clinical state is conclusive, as for other physicians it poses a number of diagnostic dilemmas. Therefore, this condition should be treated interdisciplinary. We present a case of a 40-year-old patient with a diagnosis of PG, which in the early stages of the disease was treated as an extensive phlegmon by physicians of other specialties and it presented a serious diagnostic as well as therapeutic problem.
\end{abstract}

Key words: pyoderma gangrenosum, phlegmon, inflammatory bowel disease.

\section{Introduction}

Pyoderma gangrenosum $(\mathrm{PG})$ is a relatively rare neutrophilic dermatosis, characterized by progressive skin necrosis [1, 2]. Pyoderma gangrenosum is a long-lasting disease with not fully understood etiology.

It is believed that in most cases there is an underlying disease which exacerbates PG. In the group of diseases which may be associated with PG, authors mention inflammatory bowel disease - ulcerative colitis, Crohn's disease (highest correlation), hematological disorders like monoclonal gammopathy, multiple myeloma, lymphoma, arthritis, liver disease, and autoimmune diseases like lupus erythematosus. Pyoderma gangrenosum has several variants but it usually presents as rapidly forming ulcers covered with necrotic tissue with well-defined and undermined violet borders.

Because histology findings and laboratory tests are nonspecific, proper diagnosis of PG is often problematic. Therefore, the diagnosis is based on a detailed medical history and dermatological examination which for other physicians poses a number of dilemmas so PG should be treated interdisciplinary [1-4].
The aim of this paper is to present a case of a 40-yearold male patient with PG, which was initially treated by other physicians as an extensive phlegmon with many diagnostic and therapeutic dilemmas.

\section{Case report}

A 40-year-old man (professional miner) was admitted to the Department of Dermatology, Medical University of Silesia in December 2011 with a history of extensive ulcers located within the scalp and neck. For the previous 10 years he had suffered from ulcerative colitis treated with sulfasalazine, azathioprine and currently mesalazine 4 times daily, two 500 mg tablets.

The lesions had first developed in July 2011 as small flat ulcers located around the left retroauricular skin and neck. The patient was admitted to the general surgery ward where the targeted antibiotic therapy was administered with a slight local improvement only. After the treatment the patient was discharged with a diagnosis of extensive phlegmon of the neck and scalp and blood origin abscesses of both lower limbs. In mid-November

Address for correspondence: Beata Bergler-Czop, Department of Dermatology, Medical University of Silesia, 20/24 Francuska St, 40-027 Katowice, phone/fax: +48 3225611 82, +48 322591 580, e-mail: bettina2@tlen.pl

Received: 12.10.2013, accepted: 12.11.2013. 
2011, there was a sudden exacerbation. The patient was re-admitted to the general surgery due to recurrent phlegmon of the scalp and neck with symptoms of a toxic shock. On examination, the patient had a temperature of $38^{\circ} \mathrm{C}$ without any improvement after antibiotic therapy and surgical debridement.

Cutaneous condition lead to dermatology consultations in the Department of Dermatology, Medical University of Silesia with a recommendation for a skin biopsy procedure and administration of corticosteroid therapy (methylprednisolone $32 \mathrm{mg} /$ day).

After 2 weeks, the patient was re-consulted with a diagnosis of PG confirmed by histopathological findings concomitant with ulcerative colitis. The patient was referred to the department of dermatology for further immunosuppressive therapy.

On admission, the skin examination revealed deep, painful ulcers with necrotic tissue located around the skin of the scalp and neck with well-defined borders. The ulcer edge was undermined with erythema and in duration of the surrounding skin. No lymphadenopathy was noted in the head and neck regions. Oropharynx was clear with moist mucous membranes. Capillary refill and nail beds appeared to be pink and appropriate. The laboratory tests revealed microcytic anemia, low serum iron and proteins level, high C-reactive protein (CRP) and ultrasonic determined hepatomegaly. The patient was also consulted in the department of hematology for bone marrow transplantation with a recommendation for bone marrow biopsy to exclude any lymphoproliferative process (the patient did not follow the recommendations). During hospitalization cyclosporine A was administered at a dose of $250 \mathrm{mg}$ with a gradual withdrawal of corticosteroids. After a few days of treatment a spectacular improvement had taken place (Figure 1). The patient was discharged with a recommendation for a follow-up in an outpatient clinic and tapering immunosuppressive

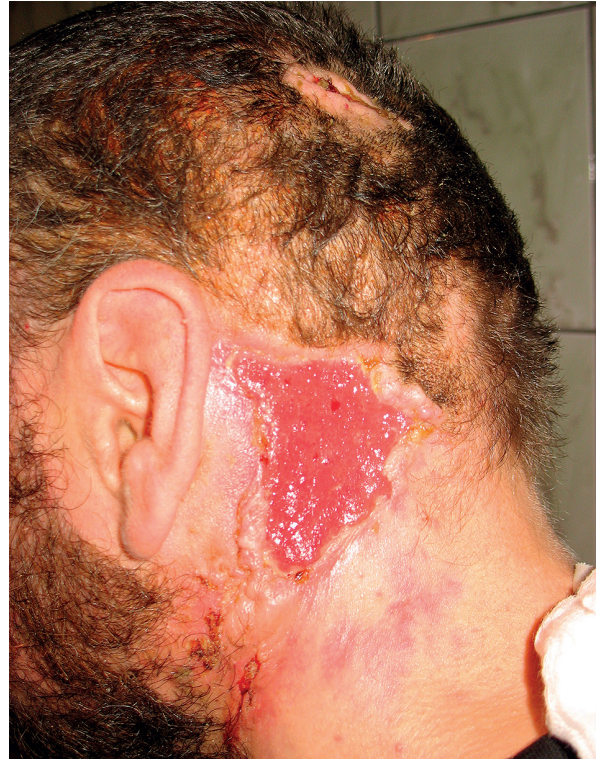

Figure 1. Ulcers located on the scalp and neck after a few weeks of treatment with CyA

therapy. On subsequent visits the local skin condition improved significantly (Figures 2 A, B) until the complete healing which took place in September 2012 (9 months' therapy). Remission of skin lesions and gastrointestinal symptoms continued until December 2012.

At the end of December 2012, the exacerbation of gastrointestinal symptoms with bloody diarrhea and low-grade fever had taken place. Treatment has been modified (mesalazine dose has been increased with mesalazine enemas administration at the same time) which resulted in a decrease in gastrointestinal symptoms. Simultaneously with the reduction of intestinal disorders new skin lesions appeared.
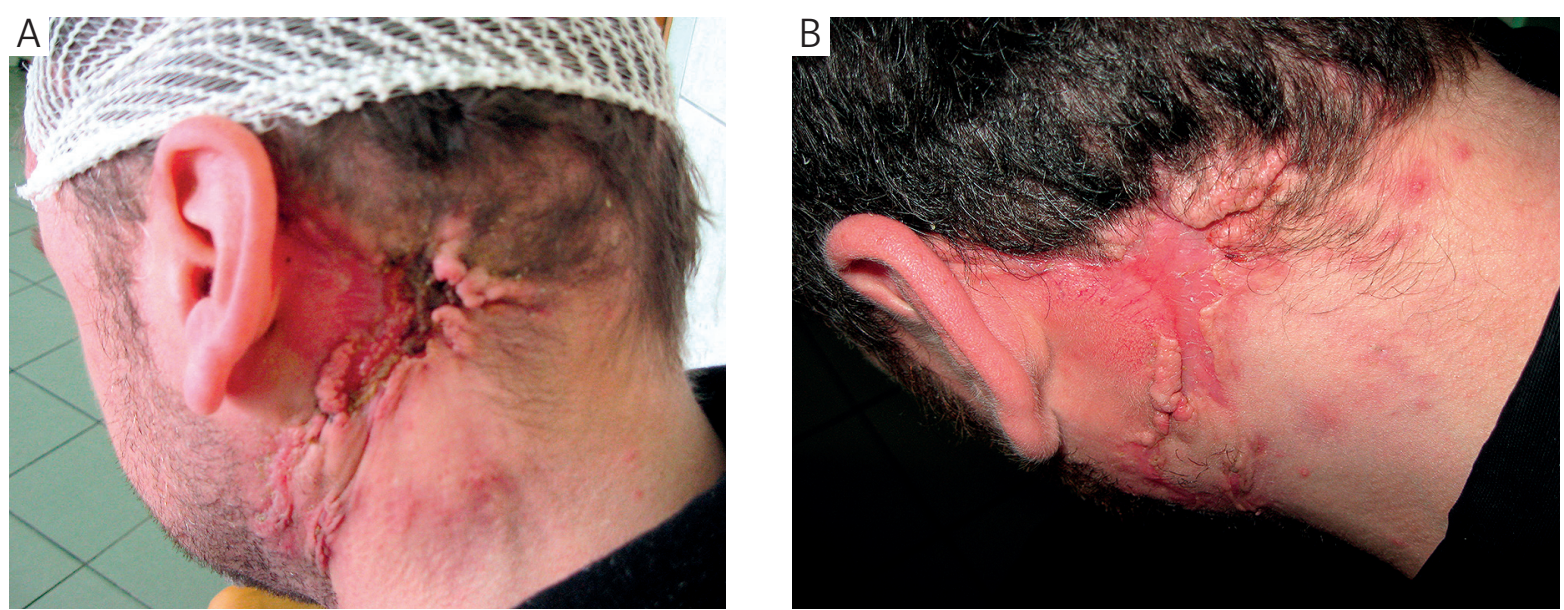

Figure 2 A, B. Further improvement of the local state in the coming months of therapy 
The patient was admitted again in January 2013 to the department of dermatology, Silesian Medical University as an emergency case due to recurrence of purulent, ulcerative lesions located over the head, neck, trunk and scrotum with a high fever. Physical examination showed a significant deterioration compared to the previous hospitalization. Lesions on the scalp and neck had the same morphology as the previous ones. New lesions appeared in the upper part of the back as indurated inflammatory nodules which after a few days broke down to form ulceration with well-defined undermined borders with a copious purulent content. Skin lesions similar to those described were present on the skin of the scrotum. Furthermore on the top of the scalp there was a soft, painful, inflamed $6 \times 4 \mathrm{~cm}$ lesion with palpable liquid fluctuant content.

The laboratory tests including erythrocyte sedimentation rate (ESR) 77, leukocytosis (a downward trend in the next studies), serum iron level 5.4 (10-30 mmol/l) Other tests like full blood count, liver and kidneys function tests, ferritin levels, electrolytes, fasting lipids, total proteins, serum protein electrophoresis and urinalysis were normal. Abdominal ultrasound - hepatomegaly. Chest X-ray - normal. Because of the previous hematologic consultation according to which proliferative disorders should be ruled out, the patient was consulted again with comments that currently there is no need for further diagnosis.

The diagnostic protocol included also a skin biopsy from the involved skin which confirmed suggested PG.

During hospitalization, intravenous antibiotics (ceftriaxone and metronidazole), corticosteroids and immunosuppressive therapy were administered which led to improvement of the general condition, normalization of body temperature without further accumulation of pus within the coatings skull. The patient was discharged in a good general condition with a recommendation to continue im-

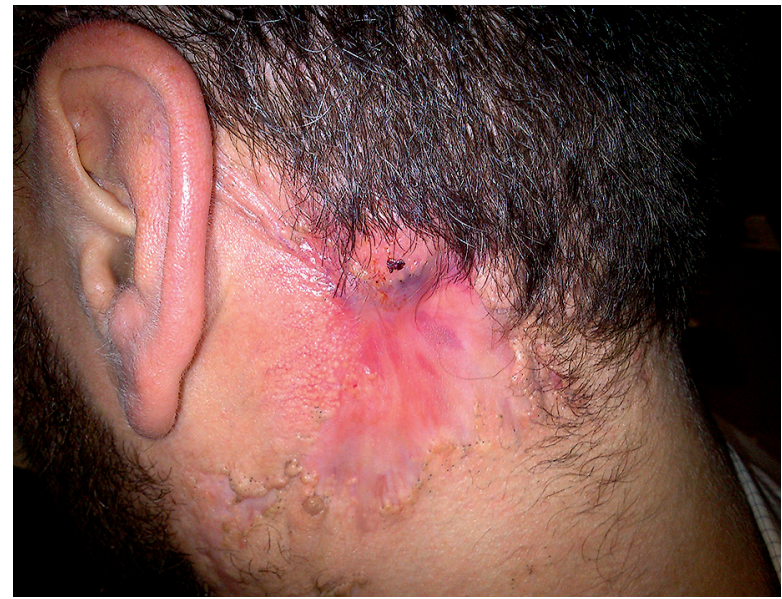

Figure 3. Remission of neck skin lesions in the second wave of the disease munosuppressive therapy - cyclosporine A at a dose of $200 \mathrm{mg} /$ day. The patient remains in the outpatient clinic with gradual remission of skin lesions, currently receives 100 mg cyclosporine A per day (Figures 3, 4).

\section{Discussion}

Pyoderma gangrenosum is characterized by the presence of sharp bordered, painful sores usually on the lower extremities. Markers of inflammation such as ESR, CRP and leukocytosis are increased.

The etiology, though not well understood, is thought to be overactive inflammatory response to various factors. The relationship between PG and underlying diseases like: inflammatory bowel diseases (mainly Crohn's disease), liver disease (hepatitis C, autoimmune hepatitis), rheumatologic and hematologic diseases (monoclonal gammopathy, leukemia) is well documented. There are also descriptions of PG after trauma (including surgical scars), accompanying sarcoidosis, solid tumors, HIV/ AIDS, conglobate and inverted acne [5-7].

In the presented case a typical relationship of skin lesions with previously diagnosed ulcerative colitis was observed. It is estimated that parenteral manifestations occur in $15-20 \%$ of patients with ulcerative colitis and $20-40 \%$ of patients with Crohn's disease. Such a frequent connection should point out to the correct diagnosis of skin lesions. Similar cases have been reported in the literature [8].

Andrade et al. [9] presented a female patient with a diagnosis of inflammatory bowel disease (anti-x-ana) with intestinal perforations and PG on the right lower extremity. Skin lesions healed after total proctocolectomy. Tanaka et al. [10] reported a case of PG after the ileo-rectal anastomosis procedure in the course of ulcerative colitis. Patvekar et al. [11] reported a rare association of PG with infectious and other intestinal diseases. In

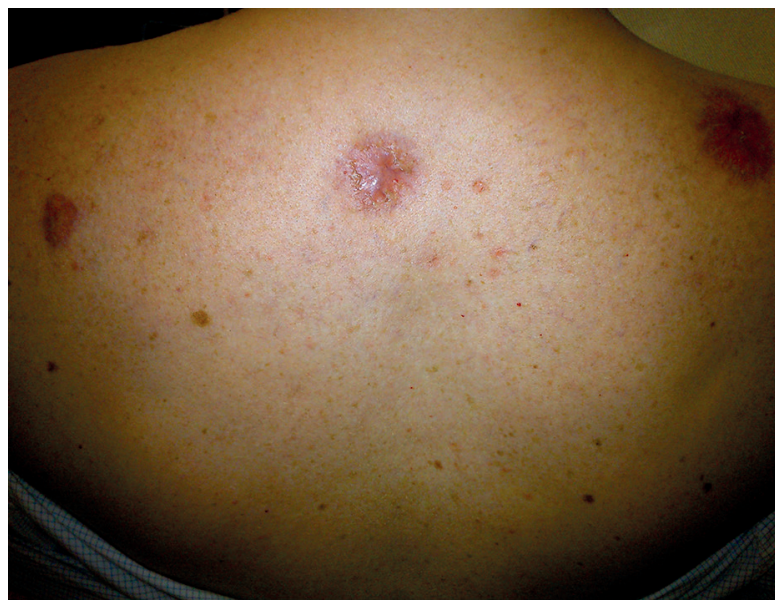

Figure 4 . The healing process located on the back after a few weeks of treatment with CyA in the relapse of the disease 
a 49-year-old man with a long history of ulcerative colitis and ilium tuberculosis, a non-healing leg ulcer appeared which was classified as PG.

Cavka et al. [12] reported necrotic right lower extremity ulcers in a 56-year-old patient. Gangrenous dermatitis was confirmed by histological evaluation. The colonoscopy showed ulceration of the colon with significant lumen stenosis. Histological examination confirmed the diagnosis of Crohn's disease.

Wu et al. [13] evaluated risk factors for peristomal gangrenous dermatitis in the abdominal region. Pyoderma gangrenosum was reported in 15 patients. Crohn's disease as an underlying disease was reported in $7 \mathrm{pa}$ tients (46.7\%), ulcerative colitis in 7 (46.7\%) and indeterminate colitis in 1 (6.7\%). Disease was active in 11 (73.3\%) patients. The authors concluded that the female gender, presence of autoimmune disorders and high body mass index are the risk factors for peristomal PG.

An association of PG with other diseases was presented in the literature as well. Because of that there is a need for a wide diagnostic process, which was planned for our patient. Unfortunately, the patient has partially failed to undergo the rest of the diagnostic process because of disobedience.

Kreuter et al. [14] reported a patient with PG and psoriasis in the course of an HIV infection. Nord et al. [15] reported a case of good therapeutic response after intravenous infusion of immunoglobulin in a 31-year-old patient with PG in the course of leukocyte adhesion deficiency.

Hinze et al. [16] reported a case of a 11-year-old boy with leukocyte adhesion deficiency type 1 and recurrent PG with superimposed fungal infection. The authors paid special attention to the diagnosis of immunodeficiency disorders especially in young patients with recurrent ulcers of the skin.

Elenberg et al. [17] presented a patient with PG after bone marrow transplantation in the course of leukocyte adhesion deficiency type 1. Bedlow et al. [18] described a case of a 5-year-old boy with a congenital deficiency of $\beta 2$ integrin which is responsible for the leukocytes adhesion as well (lad type 1) in which PG occurred.

Paller et al. [19] reported two children with an acquired immunological deficiency syndrome who developed PG. Carsuzaa et al. [20] reported a case of a 60-yearold female with PG around both ankles which appeared in the course of IgA gammopathy. Choulot et al. [21] also reported PG in a patient with IgA deficiency.

In older reports, Sánchez Yus et al. [22] reported PG coexisting with myeloma IgA lambda and congenital deficiency of cellular immunity. Barrière et al. [23] reported a case of a child with congenital hypogamma-globulinemia associated with $P G$.

Neiderer et al. [24] reported a case of a 76-year-old patient with rheumatoid arthritis associated with PG. After 9 months of topical steroids and topical tacrolimus treatment there was no improvement. After treatment with a mechanically powered negative pressure device, bioengineered cell-based product and prednisone at a dose of $40 \mathrm{mg}$, the ulcer has healed in 16 weeks.

Shenefelt [25] presented a case of a 42-year-old man with $P G$ associated with seronegative arthritis, cystic acne and hidradenitis suppurativa. Minocycline in combination with sulfasalazine was administered with a good therapeutic effect.

Pyoderma gangrenosum provides diagnostic difficulties because of the similarity to neoplastic or phlegmon lesions as in the presented case.

Wolfe et al. [26] reported a case of atypical PG of the dorsal hand which was clinically and histologically mimicking squamous cell carcinoma. Pyoderma gangrenosum mimicking squamous cell carcinoma is a rarely described subtype. Diagnosis is difficult and crucial for further treatment.

Regardless of the etiology of PG, the therapeutic process is always difficult. Treatment includes corticosteroids, dapsone, clofazimine, cyclosporine A, tacrolimus, mycophenolate mofetil, intravenous immunoglobulin, TNF- $\alpha$ inhibitors, and monoclonal antibodies [27].

After the diagnosis of PG our patient responded well to cyclosporine A therapy. However, there was a recurrence and progression of skin lesions due to an exacerbation of intestinal symptoms (despite of gastroenterological therapy).

Andrisani et al. [28] confirmed effectiveness of infliximab (TNF- $\alpha$ inhibitor) for the treatment of PG of the left chest area in a patient with associated ulcerative colitis. Also Mooij et al. [29] had a good therapeutic response in 6 patients with PG after infliximab administration.

Moschella et al. [30] have used infliximab in one case of PG associated with hidradenitis suppurativa. In this case, infliximab was used before surgical treatment of hidradenitis suppurativa. Kim and Pandya [27] have used biological treatment as well.

Traczewski and Rudnicka [31] used "off-label" adalimumab for PG treatment with a good therapeutic effect.

\section{Conclusions}

The presented case is a perfect example of how diagnostic and therapeutic problems for physicians other than dermatologists look like when facing PG which is a typical dermatology unit.

\section{Conflict of interest}

The authors declare no conflict of interest.

\section{References}

1. Jankowska-Konsur A, Maj J, Hryncewicz-Gwóźdź A. Pyoderma gangrenosum - retrospective study in 30 patients in Dermatology, Venereology and Allergology Clinic in Wrocław in years 2000-2010. Przegl Deramtol 2012; 99: 10-5. 
2. Lis-Święty A, Brzezińska-Wcisło L, Bergler-Czop B, Wcisło-Dziadecka D. Pyoderma gangrenosum and Sweet syndrome case report and review of literature. Przegl Dermatol 2012; 99: 20-5.

3. Braun-Falco O, Plewig G, Wolff HH, et al. Pyoderma gangrenosum. In: Dermatology. Braun-Falco O. Plewig G, Wolff HH (eds). Czelej, Lublin 2002; 858-61.

4. Duke G, Al Samaraee A, Husain A, et al. Pyoderma gangrenosum: a rare cause of breast ulceration. Ochsner J 2012; 12: 155-8.

5. Kosari P, Feldman SR. Treatment-resistant pyoderma gangrenosum. Dermatol Online J 2012; 18: 8.

6. Schadt CR, Callen JP. Management of neutrophilic dermatoses. Dermatol Ther 2012; 25: 158-72.

7. Thrash B, Patel M, Shah KR, et al. Cutaneous manifestations of gastrointestinal disease: part II. J Am Acad Dermatol Venereol 2013; 68: 211.

8. Kinjo F, Iraha A, Chinen $\mathrm{H}$, et al. Extraintestinal complications of inflammatory bowel disease. Nihon Rinsho 2012; 70: 261-5.

9. Andrade P, Brites MM, Figueiredo A. Synchronous pyoderma gangrenosum and inflammatory bowel disease, healing after total proctocolectomy. An Bras Dermatol 2012; 87: 637-9.

10. Tanaka K, Araki T, Okita Y, et al. Pyoderma gangrenosum occurring at the peri-ileal pouch-anal anastomosis in a patient with ulcerative colitis: report of a case. Surg Today 2012; 22: 134-9.

11. Patvekar MA, Virmani NC. Pyoderma gangrenosum with an underlying ulcerative colitis associated with bone tuberculosis. Indian Dermatol Online J 2013; 4: 43-6.

12. Cavka V, Situm M, Poduje S, et al. Pyoderma gangrenosum: case report. Acta Med Croatica 2012; 66: 25-8.

13. Wu XR, Mukewar S, Kiran RP, et al. Risk factors for peristomal pyoderma gangrenosum complicating inflammatory bowel disease. J Crohns Colitis 2013; 7: 171-7.

14. Kreuter A, Gambichler T, Hoffmann K, et al. Association of HIV infection, pyoderma gangrenosum and psoriasis. Acta Derm Venereol 2002; 82: 150-2.

15. Nord KM, Pappert AS, Grossman ME. Pyoderma gangrenosum-like lesions in leukocyte adhesion deficiency I treated with intravenous immunoglobulin. Pediatr Dermatol 2011; 28: 156-61.

16. Hinze CH, Lucky AW, Bove KE, et al. Leukocyte adhesion deficiency type 1 presenting with recurrent pyoderma gangrenosum and flaccid scarring. Pediatr Dermatol 2010; 27: 500-3.

17. Elenberg Y, Shani-Adir A, Hecht Y, et al. Pyoderma gangrenosum after bone marrow transplantation for leukocyte adhesion deficiency type 1. Isr Med Assoc J 2010; 12: 119-20.

18. Bedlow AJ, Davies EG, Moss AL, et al. Pyoderma gangrenosum in a child with congenital partial deficiency of leucocyte adherence glycoproteins. Br J Dermatol 1998; 139: 1064-7.

19. Paller AS, Sahn EE, Garen PD, et al. Pyoderma gangrenosum in pediatric acquired immunodeficiency syndrome. J Pediatr 1990; 117: 63-6.

20.Carsuzaa F, Pierre C, Dubegny M. Pyoderma gangrenosum and IgA gammopathy. Association with atrophic gastritis. Ann Dermatol Venereol 1989; 116: 707-13.

21. Choulot JJ, Saint Martin J. Pyoderma gangrenosum associated with IgA deficiency. Ann Pediatr (Paris) 1987; 34: 252.

22. Sánchez Yus E, Cabré J. Pyoderma gangrenosum (PG) with myeloma IgA lambda and deficiency of cellular immunity. Actas Dermosifiliogr 1980; 71: 35-40.
23. Barrière $\mathrm{H}$, Litoux $\mathrm{P}$, Stalder JF, et al. Pyoderma gangrenosum associated with congenital hypogammaglobulinemia. Ann Dermatol Venereol 1979; 106: 695-6.

24. Neiderer K, Martin B, Hoffman S, et al. A mechanically powered negative pressure device used in conjunction with a bioengineered cell-based product for the treatment of pyoderma gangrenosum: a case report. Ostomy Wound Manage 2012; 58: 44-8.

25. Shenefelt PD. Pyoderma gangrenosum associated with cystic acne and hidradenitis suppurativa controlled by adding minocycline and sulfasalazine to the treatment regimen. Cutis 1996; 57: 315-9.

26. Wolfe CM, Green WH, Cognetta AB Jr, et al. Atypical pyoderma gangrenosum of the dorsal hand mimicking squamous cell carcinoma. J Hand Surg Am 2012; 3: 1835-8.

27. Kim FS, Pandya AG. The use of etanercept in the treatment of peristomal pyoderma gangrenosum. Clin Exp Dermatol 2012; 37: 442-3.

28. Andrisani G, Guidi L, Papa A, et al. A case of pyoderma gangrenosum with ulcerative colitis treated with combined approach: infliximab and surgery. J Crohns Colitis 2012; 10: 154-61.

29. Mooij JE, van Rappard DC, Mekkes JR. Six patients with pyoderma gangrenosum successfully treated with infliximab. Int J Dermatol 2012; 18: 256-61.

30. Moschella SL. Is there a role for infliximab in the current therapy of hidradenitis suppurativa? A report of three treated cases. Int J Dermatol 2007; 46: 1287-91.

31. Traczewski P, Rudnicka L. Adalimumab in dermatology. Br J Clin Pharmacol 2008; 66: 618-25. 